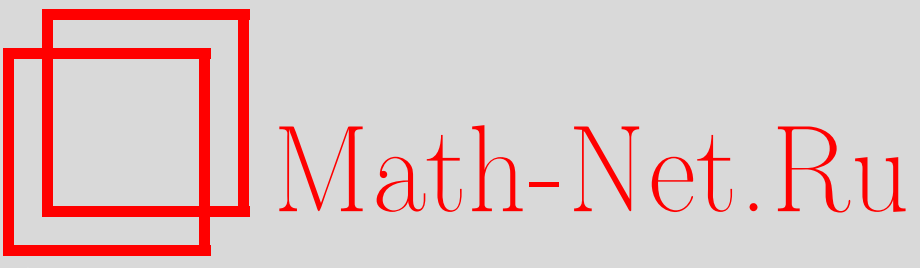

A. Yu. Vesnin, M. E. Ivanov, The polynomials of prime virtual knots of genus 1 and complexity at most 5, Sibirsk. Mat. Zh., 2020, Volume 61, Number 6, 1247-1256

DOI: https://doi.org/10.33048/smzh.2020.61.604

Use of the all-Russian mathematical portal Math-Net.Ru implies that you have read and agreed to these terms of use

http://www.mathnet.ru/eng/agreement

Download details:

IP: 52.90 .164 .192

April 26, 2023, 08:25:43 


\title{
ПОЛИНОМЫ ПРОСТЫХ ВИРТУАЛЬНЫХ УЗЛОВ РОДА ОДИН И СЛОЖНОСТИ НЕ БОЛЕЕ ПЯТИ
}

\author{
А. Ю. Веснин, М. Э. Иванов
}

\begin{abstract}
Аннотация. Простые виртуальные узлы рода один, допускающие диаграммы с не более, чем пятью классическими перекрестками, были классифицированы А. А. Акимовой и С. В. Матвеевым в 2017 г. В 2018 г. К. Каур, М. Прабхакар и А. Веснин ввели семейства $L$ - и $F$-полиномов виртуальных узлов, обобщающие аффинный индексный полином Кауффмана. В работе вводится понятие вполне плоскотривиального виртуального узла. Доказывается, что для таких узлов $L$ - и $F$ полиномы совпадают с аффинным индексными полиномом. Устанавливается, что все узлы Акимовой и Матвеева вполне плоско-тривиальны, и вычисляются их аффинные индексные полиномы.
\end{abstract}

DOI 10.33048/smzh.2020.61.604

Ключевые слова: виртуальный узел, узел в утолщенном торе, аффинный индексный полином.

\section{Введение}

Табулирование виртуальных узлов и построение их инвариантов относятся к центральным проблемам современной топологии малых размерностей. Таблицы виртуальных узлов с малым количеством классических перекрестков можно найти в [1] и на электронном ресурсе [2]. В силу эквивалентности теории виртуальных узлов и теории узлов в утолщенных поверхностях представляет интерес перечисление узлов в трехмерных многообразиях, являющихся утолщениями поверхностей заданного рода. $\mathrm{K}$ настоящему времени имеется лишь несколько результатов в этом направлении. В данной работе мы будем рассматривать простые виртуальные узлы рода один, допускающие диаграммы с малым числом классических перекрестков, которые перечислялись А. А. Акимовой и С. В. Матвеевым в [3].

Нас будет интересовать поведение некоторых полиномиальных инвариантов на классе узлов Акимовой - Матвеева. Напомним, что в [4] Кауффман определил инвариант виртуального узла - аффинный индексный полином, который обладает важными свойствами [5]. В [6] предложены обобщения аффинного индексного полинома, а именно были определены семейства полиномов $\left\{L_{K}^{n}(t, \ell)\right\}_{n=1}^{\infty}$ от переменных $t$ и $\ell$, названных $L$-полиномами, и семейство полиномов $\left\{F_{K}^{n}(t, \ell)\right\}_{n=1}^{\infty}$, названных $F$-полиномами. Ранее в [7], используя разработанные программные средства, авторы вычислили $F$-полиномы для виртуальных узлов, табулированных в $[1,2]$. В данной работе исследуются полиномиальные инварианты для узлов в утолщенном торе, построенных в [3].

Работа выполнена при поддержке лаборатории топологии и динамики Новосибирского государственного университета (грант 14.Ү26.31.0025 Министерства образования и науки Российской Федерации).

(с) 2020 Веснин А. Ю., Иванов М. Э. 

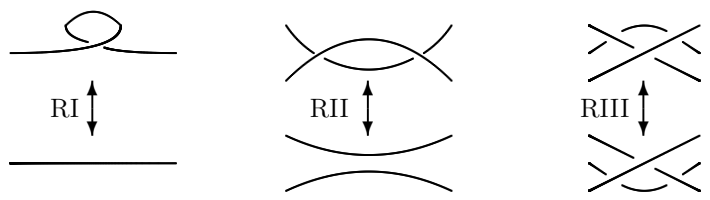

Рис. 1. Классические движения Рейдемейстера.
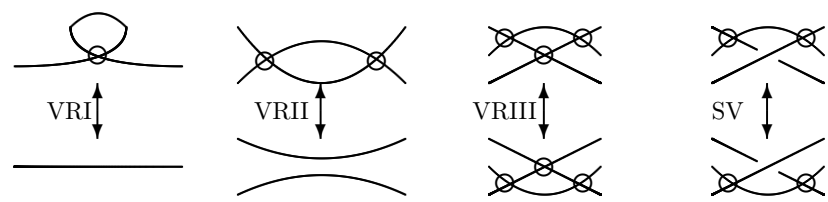

Рис. 2. Виртуальные движения Рейдемейстера.

Работа имеет следующую структуру. В разд. 1 напоминаются основные определения и факты, которые будут использованы в дальнейшем. В разд. 2 вводится понятие вполне плоско-тривиального виртуального узла и устанавливается, что для таких узлов $L$ - и $F$-полиномы сводятся к аффинному индексному полиному (лемма 2.2). В разд. 3 исследуются полиномиальные инварианты узлов Акимовой - Матвеева, диаграммы которых приведены в табл. 1 и 2. В теореме 3.1 устанавливается, что эти узлы вполне плоско-тривиальны, а в следствии 3.2 и табл. 2 приводятся их аффинные индексные полиномы. Исследование свойств узлов Акимовой - Матвеева привело авторов к следующей гипотезе, содержавшейся в первоначальной версии статьи: каждый виртуальный узел рода один является вполне плоско-тривиальным.

Авторы признательны В. В. Таркаеву, которой обратил наше внимание на то, что верность гипотезы следует из [8].

\section{1. Основные определения}

Виртуальные узлы и зацепления введены Кауффманом в [9] как естественное обобщение классических узлов. Диаграммы виртуальных узлов могут содержать как классические, так и виртуальные перекрестки. Два виртуальных узла эквивалентны тогда и только тогда, когда диаграмму одного можно перевести в диаграмму другого при помощи конечной последовательности преобразований, каждое из которых является классическим движением Рейдемейстера (движения RI, RII, RIII на рис. 1) или виртуальным движением Рейдемейстера (движения VRI, VRII, VRIII и SV на рис. 2).

Диаграмму, полученную из диаграммы виртуального узла забыванием информации о проходах/переходах в классических перекрестках, будем называть диаграммой плоского виртуального узла. Эквивалентность плоских виртуальных узлов определяется относительно плоских преобразований Рейдемейстера, которые отличаются от преобразований Рейдемейстера для виртуальных узлов тем, что забывается информация о проходах/переходах в классических перекрестках.

Пусть $D$ - диаграмма ориентированного виртуального узла. Обозначим множество классических перекрестков диаграммы $D$ через $C(D)$. Знак классического перекрестка $c \in C(D)$, обозначаемый через $\operatorname{sgn}(c)$, определяется как на рис. 3 . 


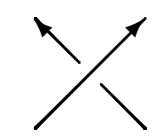

$\operatorname{sgn}(c)=+1$

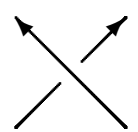

$\operatorname{sgn}(c)=-1$

Рис. 3. Знаки классических перекрестков.
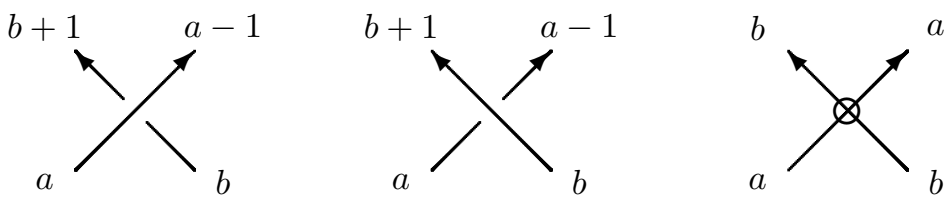

Рис. 4. Раскраска Ченга

Каждой дуге диаграммы виртуального узла сопоставим целое число так, чтобы для каждого перекрестка выполнялись соотношения, приведенные на рис. 4. В [4] Кауффман доказал, что такая раскраска диаграммы ориентированного виртуального узла, называемая раскраской Ченга, всегда существует. Действительно, дуге $\alpha$ из диаграммы $D$ можно сопоставить значение $\lambda(\alpha)=$ $\sum \operatorname{sgn}(c)$, где $O(\alpha)$ - множество таких перекрестков, которые впервые встре$c \in O(\alpha)$

чаются как переходы «над», если начинаем движение от дуги $\alpha$ и движемся в соответствии с ориентацией диаграммы.

В [10] Ченг и Гао сопоставили каждому классическому перекрестку с целочисленное значение $\operatorname{Ind}(c)$, которое можно определить следующим образом:

$$
\operatorname{Ind}(c)=\operatorname{sgn}(c)(a-b-1),
$$

где $a$ и $b$ задаются раскраской Ченга. Отметим, что раскраска Ченга не учитывает тип классического перекрестка и, по сути, строится для диаграммы плоского ориентируемого виртуального узла. Напомним, что аффинный индексный полином из [4] может быть определен следующим образом:

$$
P_{D}(t)=\sum_{c \in C(D)} \operatorname{sgn}(c)\left(t^{\operatorname{Ind}(c)}-1\right)
$$

где суммирование ведется по множеству $C(D)$ всех классических перекрестков диаграммы $D$.

В [11] Сато и Танигучи ввели понятие $n$-скрученности. Для каждого $n \in$ $\mathbb{Z} \backslash\{0\}$ определим $n$-скрученность $J_{n}(D)$ диаграммы ориентированного виртуального узла как разность числа положительных и числа отрицательных перекрестков индекса $n$. Отметим, что $J_{n}(D)$ представляет собой коэффициент при $t^{n}$ в аффинном индексном полиноме и является инвариантом ориентированного виртуального узла. Подробнее об $n$-скрученности см. в [11]. На основе $n$-скрученности в [6] определен новый инвариант $-n$-разностная скрученность $\nabla J_{n}(D):$

$$
\nabla J_{n}(D)=J_{n}(D)-J_{-n}(D)
$$

ЗАмЕчАниЕ 1.1. $\nabla J_{n}(D)$ - инвариант ориентированного виртуального узла, поскольку $J_{n}(D)$ является инвариантом ориентированного виртуального узла. Более того, $\nabla J_{n}(D)=0$ для любого классического узла.

Как показано в [6], по сути $\nabla J_{n}(D)$ отражает структуру плоского ориентируемого узла. А именно, имеет место следующий результат. 
Лемма 1.2 [6, лемма 2.4]. Для каждого $n \in \mathbb{N} n$-разностная скрученность $\nabla J_{n}(D)$ является инвариантом плоского виртуального узла.

Пусть $\bar{D}$ - диаграмма, полученная из $D$ изменением ориентации на противоположную, а $D^{*}$ - заменой перекрестков перекрестками противоположного знака.

Лемма 1.3 [6, лемма 2.5]. Пусть $D$ - диаграмма ориентированного виртуального узла. Тогда $\nabla J_{n}\left(D^{*}\right)=\nabla J_{n}(D)$ и $\nabla J_{n}(\bar{D})=-\nabla J_{n}(D)$.

Пусть $c$ - классический перекресток. Мы рассматриваем сглаживание по правилу, изображенному на рис. 5. Будем называть такое сглаживание сглаживанием против ориентации. Ориентация на $D_{c}$ индуцируется сглаживанием. Поскольку $D$ - диаграмма виртуального узлами, $D_{c}$ также диаграмма виртуального узла.

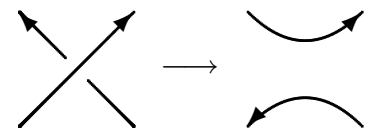

И

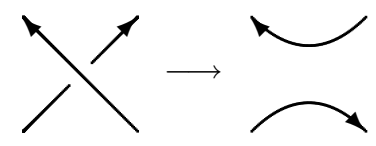

Рис. 5. Сглаживание.

ОПРЕДЕЛЕНИЕ 1.4 [6]. Пусть $D$ - диаграмма ориентированного виртуального узла $K$, а $n$ - положительное целое число. Полином $L_{K}^{n}(t, \ell)$ узла $K$ определяется следующим образом:

$$
L_{K}^{n}(t, \ell)=\sum_{c \in C(D)} \operatorname{sgn}(c)\left(t^{\operatorname{Ind}(c)} \ell^{\left|\nabla J_{n}\left(D_{c}\right)\right|}-\ell^{\left|\nabla J_{n}(D)\right|}\right) .
$$

Отметим, что $L$-полиномы обобщают аффинный индексный полином, поскольку $P_{K}(t)=L_{K}^{n}(t, 1)$ для каждого $n$.

ОПРЕДЕЛЕНИЕ 1.5 [6]. Пусть $D$ - диаграмма ориентированного виртуального узла $K$ и $n-$ положительное целое число. Тогда полином $F_{K}^{n}(t, \ell)$ определяется следующим образом:

$$
\begin{aligned}
F_{K}^{n}(t, \ell)=\sum_{c \in C(D)} \operatorname{sgn}(c) t^{\operatorname{Ind}(c)} \ell^{\nabla J_{n}\left(D_{c}\right)} & \\
& -\sum_{c \in T_{n}(D)} \operatorname{sgn}(c) \ell^{\nabla J_{n}\left(D_{c}\right)}-\sum_{c \notin T_{n}(D)} \operatorname{sgn}(c) \ell^{\nabla J_{n}(D)},
\end{aligned}
$$

где $T_{n}(D)=\left\{c \in C(D):\left|\nabla J_{n}\left(D_{c}\right)\right|=\left|\nabla J_{n}(D)\right|\right\}$.

Теорема $1.6[6]$. Для каждого целого $n \geq 1$ полиномы $L_{K}^{n}(t, \ell)$ и $F_{K}^{n}(t, \ell)$ являются инвариантами ориентированного виртуального узла.

\section{2. Вполне плоско-тривиальные виртуальные узлы}

Пусть, как и выше, $D$ - диаграмма ориентированного виртуального узла $K$, а $C(D)$ - множество всех классических перекрестков в $D$.

ОПРЕДЕЛЕНИЕ 2.1. Будем называть $D$ вполне плоско-тривиалъной, если диаграммы, полученные из $D$ и из всех $D_{c}, c \in C(D)$, забыванием информации о проходах/переходах, плоско-эквивалентны диаграмме тривиального узла. Виртуальный узел $K$ будем называть вполне плоско-тривиальным, если он обладает плоско-тривиальной диаграммой. 
Лемма 2.2. Пусть виртуальный узел $K$ вполне плоско-тривиален. Тогда

(1) для всех $n \geq 1$ имеют место $L_{K}^{n}(t, \ell)=P_{K}(t)$ и $F_{K}^{n}(t, \ell)=P_{K}(t)$;

(2) полином $P_{K}(t)$ палиндромный.

ДокАЗАтельство. (1) Пусть $D$ - вполне плоско-тривиальная диаграмма узла $K, C(D)$ - множество ее классических перекрестков и $D_{c}-$ диаграмма, полученная при сглаживании против ориентации в перекрестке $c \in C(D)$. В силу определения все указанные диаграммы плоско-эквивалентны тривиальному узлу. Ввиду леммы 1.3 имеют место равенства $\nabla J_{n}(D)=0$ и $\nabla J_{n}\left(D_{c}\right)=0$ для всех $c \in C(D)$. Подставляя эти равенства в формулы (3), (4) и сравнивая с формулой $(2)$, получаем, что $L_{K}^{n}(t, \ell)=P_{K}(t)$ и $F_{K}^{n}(t, \ell)=P_{K}(t)$.

$(2)$ Как отмечено выше, $J_{n}(D)$ является коэффициентом при $t^{n}$ в аффинном индексном полиноме. В силу равенства $\nabla J_{n}(D)=J_{n}(D)-J_{-n}(D)=0$ коэффициенты при $t^{n}$ и $t^{-n}$ совпадают, следовательно, полином $P_{K}(t)$ палиндромный.

Напомним следующее свойство аффинного индексного полинома. Обозначим через $\bar{K}$ диаграмму, полученную обращением ориентации на диаграмме $K$, а через $K^{*}$ - диаграмму, полученную заменой типа пересечения противоположным во всех классических перекрестках.

Лемма 2.3 [4]. Имеют место равенства

$$
P_{\bar{K}}(t)=P_{K}\left(t^{-1}\right) \quad \text { и } \quad P_{K^{*}}(t)=-P_{K}(t) .
$$

\section{3. Полиномиальные инварианты узлов Акимовой - Матвеева}

Простые узлы в утолщенном торе $T \times I$, произведении двумерного тора $T$ на интервал $I=[0,1]$, допускающие диаграммы с не более, чем пятью перекрестками перечислены в [3]. Общее число таких диаграмм равно 90. В силу результата Куперберга [12] это эквивалентно перечислению простых виртуальных узлов рода один, имеющих диаграммы с не более чем пятью классическими перекрестками. Для доказательства попарной различности построенных узлов авторы ввели аналог скобочного полинома Кауффмана для диаграмм, нарисованных на торе, и вычислили этот полином для всех построенных узлов. Диаграммы этих узлов на плоскости, использующие виртуальные перекрестки, приведены в [3] на рис. 17. Для удобства читателя воспроизводим эти диаграммы в табл. 3.

Теорема 3.1. Каждый узел Акимовой - Матвеева вполне плоско-тривиален.

ДокАЗАТЕЛЬСтво. Нетрудно видеть, что забывание информации о проходах/переходах в классических перекрестках диаграмм, приведенных в табл. 3, приводит к сорока различным классам плоских виртуальных узлов, у которых плоские диаграммы совпадают. Разбиение виртуальных узлов на эти классы приведено в табл. 1. Далее рассматриваются представители этих классов и для каждого из них доказывается свойство вполне плоско-тривиальности. Отметим, 
Таблица 1. Разбиение на классы

\begin{tabular}{|c|c||c|c||c|c||c|c||c|c|}
\hline & узел & & узел & & узел & & узел & & узел \\
\hline 1 & 2.1 & 9 & $4.10,4.11$ & 17 & 5.10 & 25 & $5.23,5.24$ & 33 & $5.40-5.42$ \\
\hline 2 & 3.1 & 10 & $4.12-4.14$ & 18 & 5.11 & 26 & $5.25,5.26$ & 34 & $5.43-5.46$ \\
\hline 3 & $3.2,3.3$ & 11 & $4.15-4.17$ & 19 & 5.12 & 27 & $5.27-5.29$ & 35 & $5.47-5.50$ \\
\hline 4 & 4.1 & 12 & $5.1,5.2$ & 20 & 5.13 & 28 & 5.30 & 36 & $5.51-5.53$ \\
\hline 5 & 4.2 & 13 & $5.3,5.4$ & 21 & 5.14 & 29 & 5.31 & 37 & $5.54-5.59$ \\
\hline 6 & 4.3 & 14 & 5.5 & 22 & $5.15,5.16$ & 30 & $5.32,5.33$ & 38 & $5.60-5.65$ \\
\hline 7 & $4.4,4.5$ & 15 & $5.6,5.7$ & 23 & $5.17,5.18$ & 31 & $5.34-5.37$ & 39 & $5.66-5.68$ \\
\hline 8 & $4.6-4.9$ & 16 & $5.8,5.9$ & 24 & $5.19-5.22$ & 32 & $5.38,5.39$ & 40 & 5.69 \\
\hline
\end{tabular}

Таблица 2. Полиномиальные инварианты

\begin{tabular}{|c|c|}
\hline узел $K$ & полином $P_{K}(t)$ \\
\hline $4.4,4.5,5.15,5.16,5.27,5.28,5.29,5.30$, & 0 \\
$5.31,5.45,5.47,5.48,5.67,5.69$ & $t^{-1}-2+t$ \\
\hline $\begin{array}{c}2.1,3.1,4.2,5.6,5.7^{*}, 5.10^{*}, 5.13^{*}, 5.19, \\
5.20,5.21^{*}, 5.22,5.23,5.24^{*}, 5.43,5.46\end{array}$ & $2 t^{-1}-4+2 t$ \\
\hline $4.1^{*}, 4.3,5.5,5.12,5.44$ & $t^{-2}-2+t^{2}$ \\
\hline $3.2,3.3,4.6,4.9,4.10,4.11,5.3,5.4^{*}, 5.8,5.9^{*}$, & $2 t^{-2}-4+2 t^{2}$ \\
\hline $5.14,5.17,5.18^{*}, 5.32,5.33,5.34,5.37,5.49,5.66$ & $t^{-2}-t^{-1}-t+t^{2}$ \\
\hline $5.1,5.2,5.11,5.25,5.26,5.50,5.68$ & $t^{-2}+t^{-1}-4+t+t^{2}$ \\
\hline $4.8,5.35^{*}, 5.39^{*}$ & $t^{-3}-2+t^{3}$ \\
\hline $4.7,5.36,5.38$ & $t^{-3}-t^{-1}-t+t^{3}$ \\
\hline $4.13,4.15,4.16,4.17,5.40,5.41,5.42^{*}, 5.52,5.53^{*}$ & $t^{-3}+t^{-1}-4+t+t^{3}$ \\
\hline 4.14 & $t^{-4}-2+t^{4}$ \\
\hline $4.12,5.51$ & $t^{-4}-t^{-2}-t^{2}+t^{4}$ \\
\hline $5.54,5.57,5.60,5.61,5.62,5.63,5.64^{*}, 5.65$ & $t^{-4}+t^{-2}-4+t^{2}+t^{4}$ \\
\hline $5.56,5.68^{*}$ & \\
\hline $5.55,5.59$ & \\
\hline
\end{tabular}

что смена типа пересечения в классическом перекрестке приведет при сглаживании против ориентации лишь к тому, что у полученного узла поменяется ориентация. Таким образом, для доказательства вполне плоско-тривиальности всех 90 виртуальных узлов достаточно рассмотреть лишь 40 представителей классов эквивалентности из табл. 1.

В качестве примера рассмотрим виртуальный узел $K=5.17$, представляющий класс эквивалентности под номером 23, изображенный на рис. 6. Забывая типы пересечений в классических перекрестках, получим диаграмму плоского виртуального узла. Применяя плоские аналоги преобразований Рейдемейстера RII, RI и преобразования VRII и VRI, нетрудно убедиться, что плоская версия диаграммы, приведенной на рис. 6 , плоско-эквивалентна диаграмме тривиального узла.

Удостоверимся, что и диаграмма, и полученные сглаживания против ориентации в классических перекрестках также плоско-эквивалентны тривиально- 
Таблица 3. Диаграмма узлов Акимовой - Матвеева

(1)




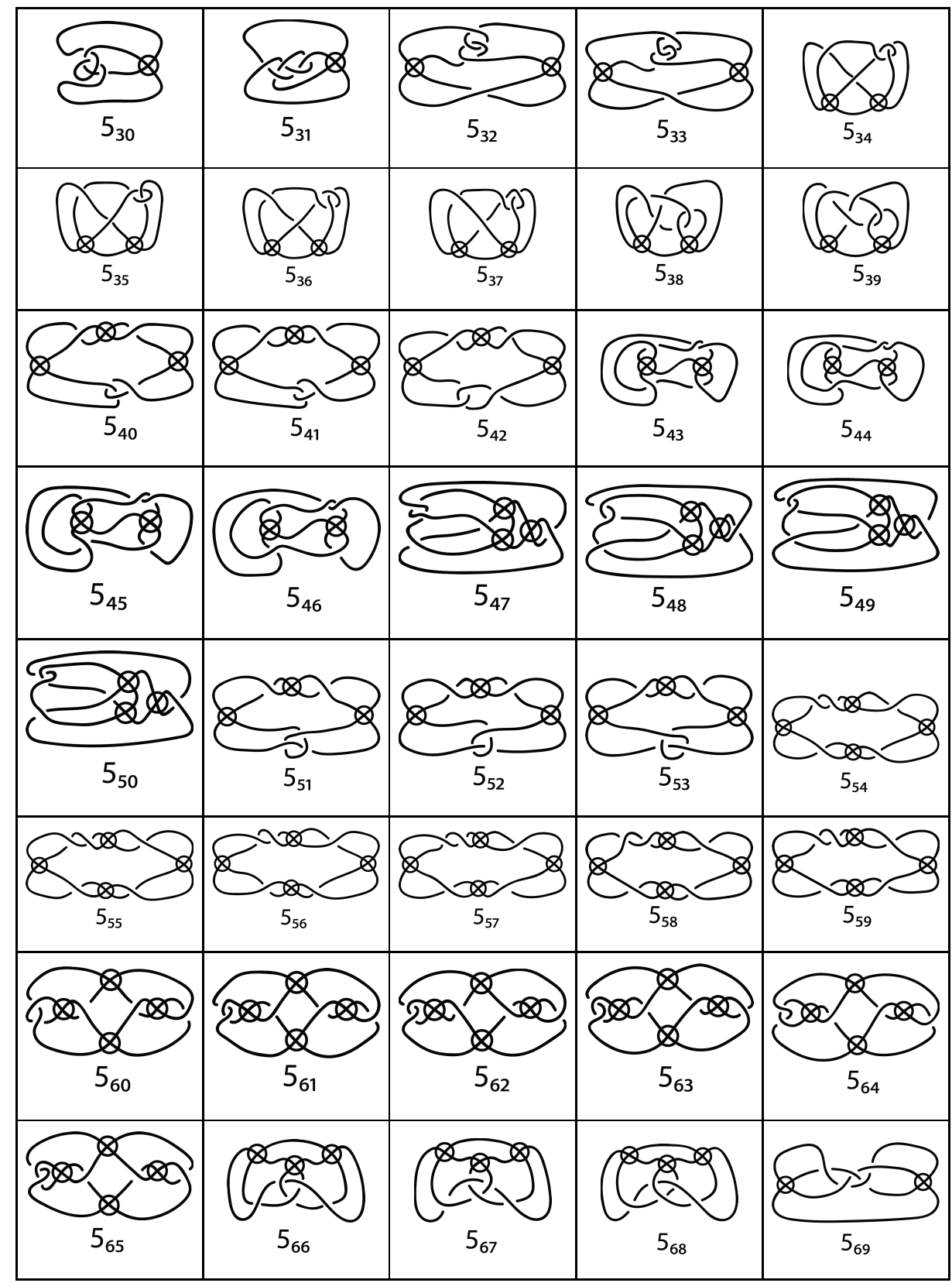

му узлу. В самом деле, диаграмма узла $K=5.17$ имеет пять классических перекрестков, обозначенных через $a_{1}, a_{2}, b_{1}, b_{2}, c$. Диаграммы, получаемые сглаживаниями в точках $a_{1}, b_{2}$ и $c$ приведены на рис. 7. Нетрудно убедиться непосредственно, что каждая из диаграмм, приведенных на рис. 7 , является диаграммой виртуального узла, плоско-эквивалентного тривиальному. Аналогично диаграммы, полученные сглаживанием против ориентации в перекрестках $a_{2}$ и $b_{2}$, также плоско-эквивалентны диаграмме тривиального узла. Таким 


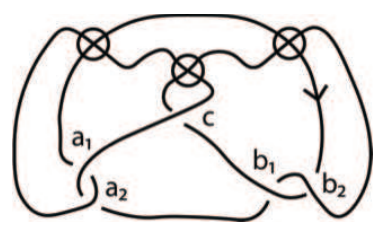

Рис. 6. Диаграмма виртуального узла 5.17.
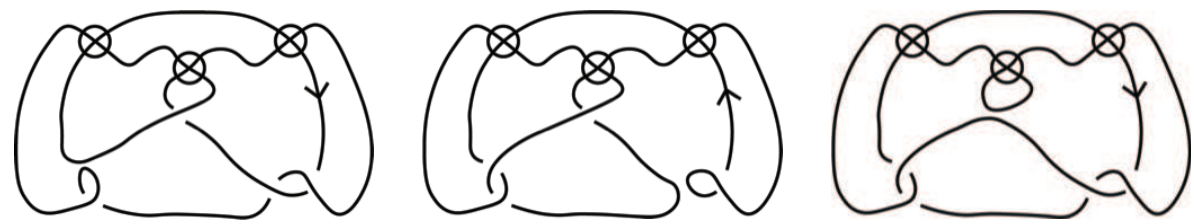

Рис. 7. Диаграммы, полученные сглаживаниями в перекрестках $a_{1}, b_{1}$ и $c$ соответственно.

образом, виртуальный узел $K=5.17$ является вполне плоско-тривиальным узлом.

Аналогичные рассмотрения, проведенные для виртуальных узлов, представляющих остальные классы эквивалентности, показывают, что все они являются вполне плоско-тривиальными, а следовательно, и все узлы Акимовой Матвеева вполне плоско-тривиальны.

Теорема 3.1 и лемма 2.2 позволяют установить следующие свойства $L$-, $F$ полиномов и аффинного индексного полинома для узлов Акимовой - Матвеева.

Следствие 3.2. Пусть $K-$ узел рода один, допускающий диаграмму $c$ не более, чем пятью двойными точками. Тогда для каждого $n \geq 1$ его $L$ - и $F$-полиномы совпадают с аффинным индексным полиномом, приведенным в табл. 2, где узлы сгруппированы в соответствии со значением полинома для узла $K$ или его зеркального образа $K^{*}$.

\section{ЛИТЕРАТУРА}

1. Dye H. An invitation to knot theory: virtual and classical. New York: Chapman and Hall/CRC, 2016. https://doi.org/10.1201/9781315370750.

2. Green J. A table of virtual knots. https://www.math.toronto.edu /drorbn/Students/GreenJ/ last updated August 10, 2004.

3. Akimova A. A., Matveev S. V. Classification of genus 1 virtual knots having at most five classical crossings // J. Knot Theory Ramifications. 2014. V. 23, N 6.1450031 (19 p.).

4. Kauffman $L$. An affine index polynomial invariant of virtual knots // J. Knot Theory Ramifications 2013. V. 22, N 04. 1340007.

5. Kauffman L. Virtual knot cobordism and the affine index polynomial // J. Knot Theory Ramifications. 2018. V. 27, N 11. 1843017.

6. Kaur K., Prabhakar M., Vesnin A. Two-variable polynomial invariants of virtual knots arising from flat virtual knot invariants // J. Knot Theory Ramifications. 2018. V. 27, N 13. 1842015.

7. Ivanov M., Vesnin A. F-polynomials of tabulated virtual knots. J. Knot Theory Ramifications. 2020. V. 29, N 8. 2050054.

8. Turaev V. Virtual strings // Ann. Inst. Fourier, Grenoble. 2004. V. 54, N 7. P. 2455-2525.

9. Kauffman L. Virtual knot theory // Europ. J. Comb. 1999. V. 20, N 7. P. 663-691.

10. Cheng Z., Gao H. A polynomial invariant of virtual links // J. Knot Theory Ramifications. 2013. V. 22. N 12. 1341002.

11. Satoh S., Taniguchi K. The writhes of a virtual knot// Fund. Math. 2014. V. 225. P. 327-341. 
12. Kuperberg G. What is a virtual knot? // Algebr. Geom. Topol. 2003. V. 3. P. 587-591.

Поступила в редакиию 6 августа 2019 г.

После доработки 6 августа 2019 г.

Принята к публикации 18 октября 2019 г.

Веснин Андрей Юрьевич

Новосибирский государственный университет,

лаборатория топологии и динамики,

ул. Пирогова, 1, Новосибирск 630090;

Институт математики им. С. Л. Соболева СО РАН,

пр. Академика Коптюга, 4, Новосибирск 630090;

Томский государственный университет,

пр. Ленина, 36, Томск 634050

vesnin@math.nsc.ru

Иванов Максим Эдуардович

Новосибирский государственный университет,

лаборатория топологии и динамики,

ул. Пирогова, 1, Новосибирск 630090

m.ivanov2@g.nsu.ru 